\title{
Post-COVID symptoms in the absence of organic deficit - Lessons from diseases we know
}

M.Sc. Dina von Werder ${ }^{1,2,3}$; Prof. Stefan Glasauer ${ }^{3}$; M.Sc. Lena Schröder ${ }^{1,2,3}$, M.Sc. Franziska Regnath $^{1}$, Dr. med. Katharina Biersack ${ }^{1}$, Prof. Peter Henningsen ${ }^{1}$, Prof. Omer Van den Bergh ${ }^{4}$; Prof. Nadine Lehnen ${ }^{1,2}$

\section{Affiliations:}

${ }^{1}$ Clinic and Policlinic for Psychosomatic Medicine and Psychotherapy, Klinikum rechts der Isar München, Technical University Munich

${ }^{2}$ Graduate School of Systemic Neurosciences, Ludwig-Maximilians-Universität München ${ }^{3}$ Computational Neuroscience, Brandenburg University of Technology Cottbus-Senftenberg ${ }^{4}$ Health Psychology, University of Leuven

\section{Corresponding author:}

Prof. Nadine Lehnen

Clinic and Policlinic for Psychosomatic Medicine and Psychotherapy

Klinikum rechts der Isar München

Technical University Munich

Ismaningerstrasse 22

81675 München

Nadine.Lehnen@mri.tum.de

Manuscript word count: 777 
While more and more patients suffer from chronic symptoms after COVID-19, a comprehensive symptom explanation is still missing. Remarkable efforts are underway to reveal structural organic impairments. This is important because symptoms can quite obviously be due to structural changes (e.g., lung, heart or brain impairments). However, they can be just as authentic and disabling when structure is intact, but information relayed from body sensors to the brain is misprocessed and misinterpreted ${ }^{1-3}$. Similar to a computer, both hardware (organ structure) and software (processing and interpretation) can fail.

Chronic symptoms in the absence of explaining structural deficits are well-known to medicine. Nowadays, many of these can be attributed to deficient information processing and interpretation, e.g., in breathlessness ${ }^{4}$ and dizziness ${ }^{5}$. This is going along with a paradigm shift in clinical medicine. It is a move away from assigning symptoms to different categories - structural and non-structural/processing - to a view in which these causes coexist. Symptoms can arise due to structural impairments or due to processing deficits, but most likely due to a combination of both. The relative influence of each component on the experienced symptom varies within each patient and can change over time to the point where there is complete decoupling of symptoms and structural impairments ${ }^{1,2}$. But whatever the contribution, once perceived, symptoms are genuine and impairing and patients deserve to have both structure and processing thoroughly and equally examined. There are established paradigms to investigate processing deficits, e.g., in breathlessness, fatigue and dizziness:

Breathlessness and fatigue. While breathlessness can arise from structural organic impairments, patients can also experience breathlessness in the absence of any structural damage. Bogaerts et $\mathrm{al}^{4}$ studied breathing behavior and perception of breathlessness in 
such patients using a rebreathing paradigm. They found characteristic alterations in breathlessness perception only apparent in certain contexts and uncoupled from breathing behavior and physiology. Similar processing deficits were found in patients with chronic fatigue and fibromyalgia without any structural impairment ${ }^{6}$.

Dizziness. Similarly, while dizziness can arise from structural, organic impairment, there is also evidence that it can manifest without it. Using an eye-head coordination paradigm, Lehnen et $\mathrm{al}^{7}$ and Schröder et $\mathrm{al}^{5}$ showed measurable head and gaze instability in patients with fully intact central and peripheral vestibular structures. This indicates aberrant sensorimotor processing. Interestingly, difficulties in motor behavior resembled those of patients with structural deficits, i.e., bilateral vestibulopathy and cerebellar ataxia. This impressively demonstrates that both structural and processing deficits play a role in symptom perception: all patient groups, with and without structural impairments, experience dizziness and, importantly, show similar and measurably altered motor behavior. Applying the rebreathing paradigm described above to patients with post-COVID breathlessness and fatigue and the eye-head coordination paradigm to patients with postCOVID dizziness has the potential to provide a measurable correlate and the basis for a diagnostic test for three important post-COVID symptoms. This might also help to understand how these symptoms arise. The theory behind this is that symptom perception is not a passive reflection of input from body sensors but is a constructive process by the brain that takes prior experiences into account, e.g., about the body, social interactions, emotions, cognitions and behavior. These prior experiences are used to build internal models, i.e., neural representations of the environment as well as the own body ${ }^{1,2}$. The following example (Adelson's Checker Shadow Illusion) shows how strongly internal models can influence perception. In Figure 1 (left side), square $A$ is perceived as darker than square 
B by most people. However, both squares are the same shade of grey, i.e., exactly the same physical information is conveyed to the eye (Figure 1, right side). Nevertheless, when looking at the whole picture this same information leads to two different perceptions. Most people experience $A$ as darker because they have experienced how shadows change the appearance of an object and integrate this information into their perception. Interestingly, explicit knowledge that both squares are the same shade of grey does not influence and 'correct' perception: even after reading this paragraph, A seems darker than B.

Especially when sensory information is incomplete, noisy or ambiguous, information processing takes existing knowledge into account to actively interpret the situation and act upon it. However, transferred to the perception of body signals, if this processing of sensory information goes wrong (e.g., when internal models are too rigid), it bares the risk for the development of symptoms while there is only weak or absent physiological dysfunction ${ }^{1,2}$. Such processing deficits might also elicit symptoms in post-COVID patients.

We propose to test whether such processing deficits are present in post-COVID symptoms that do not have a structural organic explanation. The aforementioned paradigms offer a promising starting point for this. 


\section{References}

1. Van den Bergh O, Witthöft M, Petersen S, Brown RJ. Symptoms and the body: Taking the inferential leap. Neurosci Biobehav Rev. 2017;74(Part A):185-203. doi:10.1016/j.neubiorev.2017.01.015

2. Henningsen P, Gündel H, Kop WJ, et al. Persistent physical symptoms as perceptual dysregulation: A neuropsychobehavioral model and its clinical implications. Psychosom Med. 2018;80(5):422-431. doi:10.1097/PSY.0000000000000588

3. Edwards MJ, Adams RA, Brown H, Pareés I, Friston KJ. A Bayesian account of “hysteria." Brain. 2012;135(11):3495-3512. doi:10.1093/brain/aws129

4. Bogaerts K, Van Eylen L, Li W, et al. Distorted Symptom Perception in Patients With Medically Unexplained Symptoms. J Abnorm Psychol. 2010;119(1):226-234. doi:10.1037/a0017780

5. Schröder L, von Werder D, Ramaioli C, et al. Unstable Gaze in Functional Dizziness: A Contribution to Understanding the Pathophysiology of Functional Disorders. Front Neurosci. 2021;15:685590. doi:10.3389/fnins.2021.685590

6. Van Den Houte M, Bogaerts K, Van Diest I, et al. Perception of induced dyspnea in fibromyalgia and chronic fatigue syndrome. J Psychosom Res. 2018;106:49-55. doi:10.1016/j.jpsychores.2018.01.007

7. Lehnen N, Schröder L, Henningsen P, Glasauer S, Ramaioli C. Deficient head motor control in functional dizziness: Experimental evidence of central sensory-motor dysfunction in persistent physical symptoms. Prog Brain Res. 2019;249:385-400. 
Figure 1

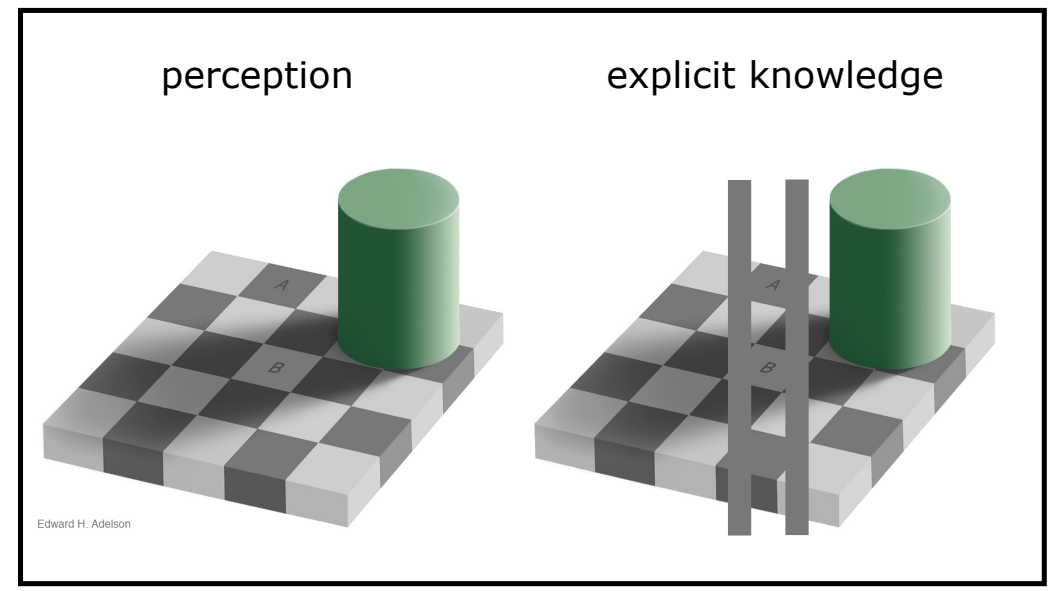

Figure 1 Adelson's Checker Shadow Illusion (adapted from Edward H. Adelson).

Most people perceive square $A$ darker than square $B$ (left side). However, square $B$ is just an identical copy of square $A$ (right side), illustrating that the brain may construct different perceptions with exactly the same physical input. 